\title{
Chapter 5 \\ Provider Workload and Multiple \\ Morbidities in the Caribbean and South Africa
}

Bilikisu R. Elewonibi, Shalini Pooransingh, Natalie Greaves, Linda Skaal, Tolu Oni, Madhuvanti M. Murphy, T. Alafia Samuels, and Rhonda BeLue

\subsection{Introduction}

Africa and the Caribbean have the highest prevalence of HIV/AIDS in the world and are also challenged by a chronic non-communicable disease (NCD) epidemic. Although South Africa is an upper- middle income country, and the Caribbean is largely a mix of middle and high income countries, with Haiti the only low income country, their health systems are not fully capable of meeting all the needs of

\footnotetext{
The original version of this chapter was revised. An erratum to this chapter can be found at https://doi.org/10.1007/978-3-319-77685-9_10

B. R. Elewonibi $(\bowtie)$

Department of Global Health and Population, Harvard T.H.Chan School of Public Health, Boston, MA, USA

S. Pooransingh

Faculty of Medical Sciences, The University of the West Indies,

St Augustine, Trinidad and Tobago

N. Greaves · T. A. Samuels

The George Alleyne Chronic Disease Research Centre, The University of the West Indies, Barbados

L. Skaal

Department of Public Health, University of Limpopo, Polokwane, South Africa

T. Oni

Department of Public Health and Family Medicine, University of Cape Town, Cape Town, South Africa

M. M. Murphy

Faculty of Medical Sciences, The University of the West Indies, Barbados

R. BeLue

College for Public Health and Social Justice, Saint Louis Universtiy, St. Louis, MO, USA
} 
patients with comorbidities. In many middle and high-income countries, a dedicated system of care for people living with HIV/AIDS (PLWHAs) was developed in parallel to existing systems of care for chronic NCDs, and these lack an integrated approach. The success of the HIV/AIDS care model has been attributed to increased advocacy, political will, country leadership, empowerment of health workers, increased community involvement and donor funding. These inputs enabled deployment of multi-disciplinary teams, task-shifting, support for adherence via family and community, along with monitoring and evaluation, health system strengthening and continuity of care. Currently, the workload, as well as policies and practices required to manage HIV and NCD care from a patient and health care systems perspective in the Caribbean and South Africa, are not fully known.

This chapter discusses the current state of health care for HIV and NCDs in the Caribbean and South Africa and presents two case studies that examine the challenges faced by patients who need to negotiate these two health care systems for their co-morbid conditions. The cases presented examine the complexity of care for persons living with HIV and Type 2 Diabetes Mellitus (T2DM) living in South Africa and in the Caribbean Islands of Trinidad and Barbados using a modified version of the Cumulative Complexity Model (CCM). This model posits that as the burden of disease and resulting workload increases, the patient capacity to respond to it diminishes.

\subsection{Background}

\subsubsection{HIV and Noncommunicable Disease (NCD) Care in South Africa}

South Africa has the largest antiretroviral treatment (ART) program in the world (Dalal et al. 2011; Levitt et al. 2011; Mayosi et al. 2012). As a result of new guidelines for treatment initiation, aggressive intervention and almost full medical coverage have contributed to the progress in prevention of mother-to-child transmission (pMTCT) programs (Johnson 2012; Levitt et al. 2011; Mayosi et al. 2012; World Health Organization 2011). More than $98 \%$ of women receive an HIV test during pregnancy and $91.7 \%$ of HIV-positive mothers are receiving ART or prophylaxis (Requejo et al. 2012). However, challenges remain, including access to HIV treatment for pregnant women due to stock depletion, further pMTCT due to a lack of feeding support in the postnatal period, and ensuring (Becquet et al. 2009) expansion of treatment to the individuals who still need ART (Mayosi et al. 2012). The program has also struggled with treatment cost, availability of drug suppliers, and sustaining the population (because of logistics in administering medication to a large population, or administration/operation challenges) (Mayosi et al. 2012). There is inadequate data on the longterm consequences of HIV infection and treatment in South Africa. Discrimination and social marginalization continue to be experienced daily by people who are affected by HIV (Mayosi et al. 2012). These social exclusions further contribute to reluctance to test for HIV and to visit dedicated HIV treatment sites. 
Most patients in South Africa with NCDs are managed at the primary health care level (Levitt et al. 2011). Major weaknesses of the current structure center around continuity of care, comprehensive care, consistent drug supply, and regular auditing of quality of care (Dalal et al. 2011; Dawson et al. 2013; Levitt et al. 2011; Oti 2013). Support from community health workers for patients with NCDs at the primary care level is rarely available; there is seldom opportunity for patient education, and health workers lack communication skills, which results in suboptimal patient-centered care (Levitt et al. 2011). In addition, there are shortages of essential drugs, lack of access to other drugs, budget constraints that prevent health providers from ordering essential tests, lack of recall systems for non-attenders, and inadequate patient records (Levitt 2008; Levitt et al. 2011). Consequently, patients with NCDs who attend primary care services seldom achieve an adequate level of care.

\subsubsection{HIV Care in the Caribbean}

In 2004, Trinidad and Tobago (TT) established a National AIDS Coordination Committee (NACC) to coordinate and monitor implementation of the national strategic plan for HIV/AIDS (Duke et al. 2010). The Ministry's National AIDS Programme was renamed the HIV and AIDS Coordinating Unit (HACU) in 2006. One of the priorities for TT was to expand access to HIV testing and in 2006, the Ministry of Health established a same-visit HIV testing program (Laptiste et al. 2013). This program provides confidential testing that confidential testing that does not require laboratory confirmation, providing clients with HIV status information in a single visit. Another successful program is the Rap Port, a youth drop-in center targeting the age group 13-25 years. It was created in response to the epidemic of HIV/AIDS/STIs affecting this population. Its goal was to prompt healthy lifestyle practices by creating a supportive environment (Laptiste et al. 2013). Additionally, the government's prevention of mother-to-child transmission (PMTCT) program was implemented in 1999 in Tobago and 2000 in Trinidad (Laptiste et al. 2013). By 2009 , approximately $96.7 \%$ of all women attending public antenatal clinics throughout Trinidad and Tobago participated in the program (Laptiste et al. 2013). In 2008, dried blood spot testing for infants was introduced. Antiretroviral therapy is available free of charge at government hospitals and at seven locations throughout the country (Laptiste et al. 2013).

In Barbados, the government provides comprehensive health care, free at the point of delivery, to all its citizens. With the availability of highly active antiretroviral therapy (HAART) since 2002 and the provision of free care and treatment to all HIVinfected persons in Barbados, there has been a consistent decline in the HIV-specific death rate (Kumar et al. 2006, 2007). All HIV-infected persons receive their inpatient care at Queen Elizabeth Hospital and ambulatory care is provided by a centralized HIV clinic that has operated from Ladymeade Reference Unit since 2002 (Kumar et al. 2006). The Ladymeade Reference Unit was established as part of the Government's expanded response to the HIV epidemic and consists of a clinic, inhouse pharmacy and an internationally accredited laboratory (Kumar et al. 2006; 
Landis et al. 2013). Queen Elizabeth Hospital is the only public hospital in Barbados, providing more than $95 \%$ of all inpatient care to the population (Kumar et al. 2007). Barbados also has a well-organized Pediatric HIV Surveillance Program that monitors mother-to-child HIV transmission (Kumar et al. 2004; Kumar and Bent 2003). Since 1990, voluntary antenatal HIV screening, and pre- and post-test counseling has been offered to all pregnant women irrespective of previously known HIV antibody status (Kumar and Bent 2003). Despite easy availability of treatment and care, late presentation has been a major cause of concern (Kilaru et al. 2004, p 4) and widespread stigma and discrimination may be an important issue underlying this problem (Rutledge and Abell 2005).

\subsubsection{Non-communicable Disease (NCD) in the Caribbean}

The small island developing states (SIDS) in the Caribbean islands face significant challenges with the worst NCD epidemic in the region of the Americas. Premature mortality from NCDs is double the rate in North America. This is due to high prevalence of risk factors, especially obesity, and the limited resources available to address and implement NCD policies (Boda 2013). For example, in Trinidad and Tobago $78 \%$ of deaths are due to NCDs, yet there are only 8 physicians per 10,000 people [compared to 25 per 10,000 in the U.S. (Boda 2013)]. These numbers illustrate the reality of the health services strain and area major barrier within Trinidad and Tobago. There are NCD interventions in place, such as the Chronic Disease Assistance Programme (CDAP) supported by Trinidad and Tobago's Ministry of Health. CDAP provides free prescription drugs and other pharmaceutical items to individuals who suffer from diabetes, high blood pressure, cardiac diseases, among other conditions (Ramrattan 2011). However, programs like CDAP do not address the need for NCD prevention.

Heads of government of the Caribbean community (CARICOM) convened in a summit dedicated to NCDs in 2007 (Hospedales et al. 2011). Their declaration, entitled 'Uniting to Stop the Epidemic of Chronic Non-communicable Diseases' (also referred to as the Port of Spain Declaration), focused on prevention and control of NCDs through comprehensive and integrated prevention and control strategies. Each head of government gave clear policy directions for an intersectoral approach that addresses many key risk factors. As Table 5.1 illustrates, Jamaica fully implemented 17 of the 26 tasks set by the 2007 Port of Spain Declaration in 2010 (Samuels et al. 2014). Included in this progress is Jamaica's National Health Fund (NHF), an effort which subsidizes screening and prevention programs targeted at NCDs (established in 2003).

It is clear that there is more work to be done in the area of NCD prevention and control; however, CARICOM's efforts demonstrate hope for regional cooperation and collaboration against the NCD epidemic. These efforts also allow for countries to learn from each other's successes and areas of concern. 
Table 5.1 Compliance with 26 indicators from the Port-of-Spain

\begin{tabular}{l|c|c}
\hline Highest compliers & 20 & $77 \%$ \\
\hline Barbados & 19 & $73 \%$ \\
\hline Trinidad and Tobago & 18 & $69 \%$ \\
\hline Jamaica & 17 & $65 \%$ \\
\hline Bahamas & & \\
\hline Middle compliers & 15 & $58 \%$ \\
\hline $\begin{array}{l}\text { Grenada, Cayman Islands, } \\
\text { Guyana, St. Lucia }\end{array}$ & & \\
\hline Suriname, Antigua \& Barbuda & 14 & $54 \%$ \\
\hline Bermuda & 12 & $46 \%$ \\
\hline British Virgin Islands, Dominica & 11 & $42 \%$ \\
\hline Belize & 9 & $35 \%$ \\
\hline St. Kitts and Nevis & 8 & $31 \%$ \\
\hline St. Vincent and the Grenadines & 7 & $27 \%$ \\
\hline Lowest compliers & & \\
\hline Anguilla & 5 & $19 \%$ \\
\hline Turks and Caicos & 2 & $8 \%$ \\
\hline Montserrat & 2 & $8 \%$ \\
\hline Haiti & 1 & $4 \%$ \\
\hline $\begin{array}{l}\text { Declaration for CARICOM Countries } \\
\text { score }\end{array}$ & 2014 \\
\hline
\end{tabular}

\subsection{Case Studies Examining Patient Challenges Associated with Multiple Morbidity}

The case studies presented from South Africa and the Caribbean used a qualitative approach to explore and examine the perspectives and experiences of patients related to workload associated with, and capacity required to manage, HIV/NCD co-morbidity as they negotiate parallel systems of healthcare for HIV and for T2DM. Managing comorbid conditions requires considerable time, effort and money, which add distress to patients who are already burdened by their illnesses. A novel theoretical framework, Shippee's Cumulative Complexity Model, was used to explore how individuals cope with these demands. Phase one of the study was conducted in South Africa and then replicated in the Caribbean, which allowed us to understand workload related to HIV/NCD comorbidity across countries and regions with various income levels, stages of urbanization and health care systems.

\subsection{The Cumulative Complexity Model (CCM)}

The Cumulative Complexity Model outlines relations between the work delegated by healthcare systems to patients (their burden of treatment), and the ways in which they can balance these burdens with capacity to meet the demands of delegated work. It seeks to summarize the effects of social and clinical factors on patient outcomes 


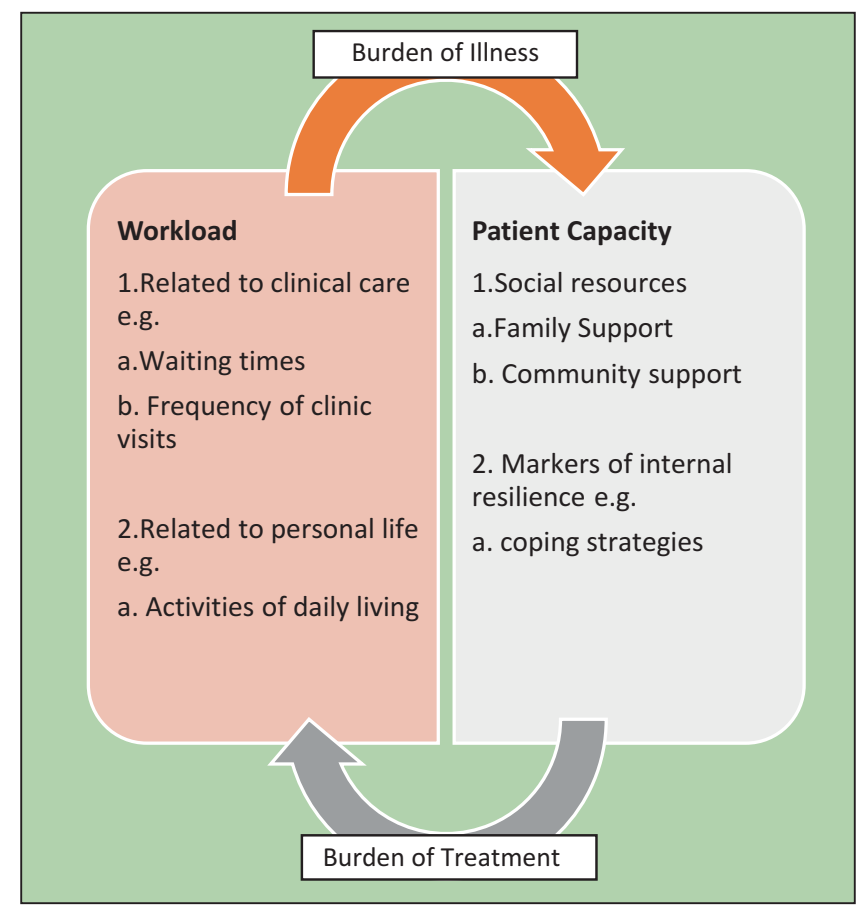

Fig. 5.1 Modified Shippee's Cumulative Complexity Model used in this study on workload in $\mathrm{HIV} / 2 \mathrm{TDM}$

(Shippee et al. 2012). It articulates that a patient's clinical outcomes are influenced by the interaction between two parameters: workload and patient capacity. In the CCM, workload refers to those factors which place a demand on a patient's "time and energy", e.g. employment and self-care activities, while patient capacity relates to the patient's ability to "handle work". Importantly, patient capacity includes social capital measures such as financial and social resources as well as physical domains such as functional morbidity (Shippee et al. 2012). It is important to note that the CCM is dynamic with complexity of care being driven not only by the primary interaction between workload and patient capacity but also by external drivers of the burden associated with illness and treatment. The domains examined in this study are represented in the modified Shippee's model shown in Fig. 5.1.

\subsection{Methodology}

\subsubsection{Data}

Data for these studies were collected through in-person interviews. Constructs explored reflected the proposed theoretical model on cumulative complexity. Specifically, interviews included questions on (i) workload: the demands on patient's 
time and energy, the demands of treatment, self-care, other comorbidities, access to care for HIV and T2DM treatment, employment status, and family responsibilities and (ii) concerns about the capacity to cope with the workload: financial and social resources, health literacy and morbidity as it affects ability to function. The audiotaped interviews were transcribed verbatim. Random samples of translations in South Africa were periodically checked for accuracy by a third party within the research team. In South Africa, interviews were conducted in English or a local language by a native speaker and then translated back into English for analysis. In the Caribbean, all interviews were conducted in English.

\subsubsection{Setting and Sample Size}

In South Africa, the study was conducted in two settings. In the Western Cape, the study was conducted in Khayelitsha, a predominantly black African, peri-urban largely informal settlement in Cape Town. The area is largely made up of Xhosa speaking community with about two-thirds of the community employed but live in low-income households. In the Limpopo province, the study was conducted in the Mankweng district, a rural province in South Africa. Most people in Mankweng district are Sotho. Mankweng is made up of peri-urban townships, tribal villages and informal settlements, where large families are living in deprivation, lacking satisfactory water supply, sanitation and adequate access to basic services (Ellison et al. 1996; Statistics South Africa 2014). A total of 10 persons living with multiple morbidity were recruited in Cape Town and 11 in Limpopo.

The Caribbean case study took place in Barbados and in Trinidad and Tobago. Barbados has a population of approximately 280,000 persons, predominantly of the African diaspora; Trinidad and Tobago has a population of approximately 1.4 million persons with two ethnic groups predominating - persons of the East Indian diaspora and those of the African diaspora. Both settings in this case study were urban due to the location of the clinics. This resulted in 20 participants being interviewed in the Caribbean: 10 in Trinidad and 10 in Barbados.

\subsubsection{Measures}

Multi-morbidity was defined as HIV plus diagnosed T2DM, although many patients likely have additional co-morbidities. T2DM has been selected as the core NCD comorbid condition due to the complexity of its self-management. Eligible patients included those who have initiated ART and have co-morbid diabetes, in the age range of 35-65 years, as this constitutes the group with the greatest number of people who have diabetes and HIV/AIDS. Participants were recruited at the HIV clinics and approached for informed consent to participate. Participants with varied adherence histories (i.e. regular and non-regular attendees) were included. Diagnosed conditions were verified by medical record review after consent was given. 


\subsection{Data Analysis}

The data analysis team was made up of researchers from each research site. Data analysis was done using an iterative process beginning during the data collection phase. Condensed text was abstracted into increasingly higher order headings, creating codes, categories and themes. Qualitative software was used to organize and manage the data analysis. A coding scheme was developed by the research team. Each transcript was coded by one researcher, and then a second researcher independently coded for each researcher's first two transcripts to ensure coding reliability across the team (in South Africa). An analysis group determined the emerging themes from the data.

\subsubsection{Results from Case Study 1: Patient Workload and Capacity for Managing HIV/T2DM in Cape Town and Limpopo}

Perceived Patient Workload Patient workload from managing two chronic, relapsing illnesses emerged as a main theme among participants. Patients felt that they had disease management related workload from both negotiating the healthcare system and actual self-care. Patients reported that they experienced clinic-related workload including barriers related to access to care. For example, patients had to negotiate two separate clinics for HIV and T2DM. They also experienced long waiting periods and shortages of healthcare workers, most notably at the diabetes clinic. Self-care related workloads included adherence to nutritional requirements for both conditions, pill burden, engaging in physical activity, ensuring personal safety and dealing with stigma, especially for the HIV diagnosis.

Patient Capacity Patient capacity emerged as a common theme, which included both enablers and challenges to managing diabetes and HIV. Factors related to patient capacity included having a positive attitude about treatment and outcomes, having confidence in medical treatment, having support from family, having strong beliefs in religious or cultural practices, having a reasonable level of health literacy and having financial social support from their family and clinic staff.

Health System There is lack of capacity to provide drugs by the clinics or hospitals due to inventory shortages. The health system is overloaded with chronic patient admissions and frequent visits. Additionally, the health system needs skilled health care practitioners who understand dual drug interactions and side effects so they can communicate these to patients, thereby avoiding late reporting of side-effects and/ or medication noncompliance. Among the challenges cited were the attitude of health care practitioners and the tendency of rural patients to seek services of traditional healers and prophets before presenting at primary health care institutions. 


\subsubsection{Results from Case Study 2: Patient Workload and Capacity for Managing HIV/T2DM in Barbados and Trinidad \& Tobago}

The three themes which were found to predominate in this setting are described below. Emerging themes were similar in both sites.

Heath Service Provision Relating to the category of "patient workload of demands", non-personal factors such as the processes involved in heath service provision (e.g. wait times) were found to affect a person's ability to manage their illness and non-illness related tasks more so than family and self-care demands. Patients who require blood tests need to arrive at the clinics early since blood tests are only available for a limited period in the day and for those with an appointment later in the day, this would mean being at the clinic all day. For employed patients attending weekday clinics, this could result in lost wages; an evening or a Saturday clinic would be more accessible and economical for this group, but is not currently available.

Privacy and Dignity Patients with a diagnosis of HIV attending appointments for diabetes management expressed concerns around confidentiality of their medical information. They also indicated that ideally, patients should not be able to see the other patients who attend the dedicated HIV clinic in order to keep their diagnosis private and confidential.

Self-Care and Stigma HIV was considered easier to manage than diabetes. Diabetes management was viewed as much more time intensive with meal preparation time, need for a special diet, exercise, medication and blood glucose testing all being part of the care plan, which makes adherence to a management plan more complex and at times more fearful. Comparatively, HIV management was considered straightforward — "take your meds and go." However, for HIV, there was more discussion around capacity to cope, which was intertwined with experiences of stigma and discrimination. HIV was perceived to present less physical distress or "non-psychological" challenges, but higher mental/emotional challenges compared to diabetes, due to stigma associated with HIV. Diabetes, on the other hand, presented more physical/non-psychological challenges related to disease management as it impacted quality of life, but fewer mental/emotional challenges. Priority areas around diabetes care centred on managing the illness; for HIV the main concerns were related to social determinants (e.g. housing issues, employment, food insecurity), which are linked to stigma and discrimination.

As previously stated, HIV services in Barbados are centralized in a vertical programme capable of addressing the non-HIV chronic care needs of clients, while in Trinidad services are decentralized throughout the clinic system (in addition to a centralised HIV clinic). Interestingly, in Barbados it seems that while episodes of discrimination have been associated with a centralised treatment system, patient 
preference is for centralised care. In Trinidad and Tobago, participants also preferred a centralized location for their services. All participants desired institutions offering HIV services to take a holistic approach to treatment.

\subsection{Discussion}

Although the case studies were carried out in separate regions with different sociodemographic characteristics, common themes emerged. The main theme was that health service provision does not adequately cater to patients with HIV and multiple morbidities. Although this health system setup is fairly common, it subjects a person with HIV to community scrutiny, leading to adverse psychological impact (or emotional distress, or feelings of shame and isolation - not sure how specific the data is). A unique theme was observed in the second case study whereby a positive attitude, strong religious beliefs, family support and a reasonable level of health literacy led to adjustment over time. This theme was not present in the first case study.

\subsection{Lessons Learned from Cross National Work}

This work was an example of successful cross country, cross region collaboration made possible through the Pan Institution Network for Global Health (PINGH). Collaboration resulted in a successful grant application among Institutions of the Network, which made the Caribbean leg of the study possible. Further, cross country collaboration resulted in the sharing of study protocols and expertise in data analysis which, in 2016, culminated in two successful pilot studies. These were used to design the Institutional Review Board proposals used across the research sites in the Caribbean. While there were no challenges in adapting the protocol to a culturally appropriate Caribbean format, the IRB process in Jamaica was not completed in time for their inclusion (or participation). Inter-island collaboration was effected through online meetings and with face-to-face project sensitization and analysis workshops. The face-to-face meetings of the research team were vital quality assurance steps and served to foster cohesiveness. The success of this collaboration was also influenced by the effective leadership of the Network.

\subsection{Implication for the Field and Future Work}

The HIV programs in developing countries take a 'vertical' approach, with singledisease prevention and treatment programs implemented by specialized health delivery services separate from routinely operated primary health care facilities. 
It is characterized by the use of additional and separate staff, registration processes, and newly constructed buildings. However, patient response and health outcomes demonstrate that a model which is patient-centered rather than disease-centered is the only truly sustainable option. To address multiple health challenges simultaneously will require an expansion of health system capacity, particularly in middleand high-income countries with economically disadvantaged subpopulations. However, existing intervention priorities of HIV/AIDS programs can be accurately applied to NCD care management and a redesign of chronic care systems. Such a coordinated or integrated approach ensures that resources are shared and maximized, bringing an overall improvement to the health system.

This study shows that increasing a patient's psychological capacity as marked by their internal resilience is fundamental to the ability to cope with dual diseases, especially when social, family and community support are diminished as a result of stigma and discrimination. It emphasizes the need for holistic approaches to chronic disease clinical care while advocating for acceptance and normalization of conditions such as HIV in the wider society.

\section{Challenges}

- Working across multiple sites requires careful timing and more resources than single-sited work. This may require a staggered study design, rather than concurrent.

- Health system capacities in low and middle income settings are limited by workload and stockouts, making implementation of recommendations difficult.

\section{Lessons Learned}

- Cross continental study sites allowed us to understand workload related to HIV/NCD comorbidity in countries with various income levels, stages of urbanization and health care systems.

- Cross country collaboration resulted in the sharing of study protocols and expertise in data analysis resulting in two successful pilot studies being accomplished in 2016.

\section{References}

Becquet R, Bland R, Leroy V et al (2009) Duration, pattern of breastfeeding and postnatal transmission of HIV: pooled analysis of individual data from west and south African cohorts. PLoS ONE 4(10):e7397. https://doi.org/10.1371/journal.pone.0007397

Boda PJ (2013) The challenge of combatting non-communicable diseases in Trinidad: access to hospital care. Health 5(11):12

Dalal S, Beunza JJ, Volmink J et al (2011) Non-communicable diseases in sub-Saharan Africa: what we know now. Int J Epidemiol 40(4):885-901. https://doi.org/10.1093/ije/dyr050 
Dawson R, Rom WN, Dheda K, Bateman ED (2013) The new epidemic of non-communicable disease in people living with the human immunodeficiency virus. Public Health Action 3(1): 4-6. https://doi.org/10.5588/pha.12.0048

Duke V, Samiel S, Musa D et al (2010) Same-visit HIV testing in Trinidad and Tobago. BMC Public Health 10(1):185. https://doi.org/10.1186/1471-2458-10-185

Ellison GT, De Wet T, IJsselmuiden CB, Richter LM (1996) Desegregating health statistics and health research in South Africa. S Afr Med J 86(10):1257-1262

Hospedales CJ, Samuels TA, Cummings R et al (2011) Raising the priority of chronic noncommunicable diseases in the Caribbean. Rev Panam Salud Publica 30(4):393-400. https://doi. org/10.1590/S1020-49892011001000014

Johnson LF (2012) Access to antiretroviral treatment in South Africa, 2004-2011. South Afr J HIV Med 13(1.) http://www.ajol.info/index.php/sajhivm/article/view/77233. Accessed 25 Oct 2017

Kilaru KR, Kumar A, Sippy N (2004) CD4 cell counts in adults with newly diagnosed HIV infection in Barbados. Rev Panam Salud Publica 16(5):302-307. https://doi.org/10.1590/ S1020-49892004001100002

Kumar A, Bent V (2003) Characteristics of HIV-infected childbearing women in Barbados. Rev Panam Salud Publica 13(1):1-9. https://doi.org/10.1590/S1020-49892003000100001

Kumar A, Rocheste E, Gibson M et al (2004) Antenatal voluntary counseling and testing for HIV in Barbados: success and barriers to implementation. Rev Panam Salud Publica 15(4):242-248. https://doi.org/10.1590/S1020-49892004000400004

Kumar A, Kilaru KR, Forde S, Roach TC (2006) Changing HIV infection-related mortality rate and causes of death among persons with HIV infection before and after the introduction of highly active antiretroviral therapy analysis of all HIV-related deaths in Barbados, 1997-2005. J Int Assoc Phys AIDS Care 5(3):109-114. https://doi.org/10.1177/1545109706288587

Kumar A, Kilaru KR, Sandiford S, Forde S (2007) Trends in the HIV related hospital admissions in the HAART era in Barbados, 2004-2006. AIDS Res Ther 4(1):4. https://doi. org/10.1186/1742-6405-4-4

Landis RC, Branch-Beckles SL, Crichlow S et al (2013) Ten year trends in community HIV viral load in Barbados: implications for treatment as prevention. PLoS One 8(3):e58590. https://doi. org/10.1371/journal.pone.0058590

Laptiste C, Beharry V, Edwards-Wescott P (2013) A review of the response to HIV/AIDS in Trinidad and Tobago: 1983-2010. SAHARA J 10(2):72-82. https://doi.org/10.1080/172903 76.2013 .869406

Levitt NS (2008) Diabetes in Africa: epidemiology, management and healthcare challenges. Heart 94(11):1376-1382. https://doi.org/10.1136/hrt.2008.147306

Levitt NS, Steyn K, Dave J, Bradshaw D (2011) Chronic noncommunicable diseases and HIVAIDS on a collision course: relevance for health care delivery, particularly in low-resource settings-insights from South Africa. Am J Clin Nutr 94(6):1690S-1696S. https://doi. org/10.3945/ajen.111.019075

Mayosi BM, Lawn JE, van Niekerk A et al (2012) Health in South Africa: changes and challenges since 2009. Lancet 380(9858):2029-2043. https://doi.org/10.1016/S0140-6736(12)61814-5

Oti SO (2013) HIV and noncommunicable diseases: a case for health system building. Curr Opin HIV AIDS 8(1):65-69. https://doi.org/10.1097/COH.0b013e32835b8088

Ramrattan D (2011) A preventative approach to healthcare (chronic non-communicable diseases) in Trinidad and Tobago. WJSTSD 8(4):339-359

Requejo J, Bryce J, Victora C (2012) Countdown to 2015 maternal, newborn and child survival: building a future for women and children: the 2012 report. In Countdown to 2015 maternal, newborn and child survival: building a future for women and children: the 2012 report (pp. 56-56)

Rutledge SE, Abell N (2005) Awareness, acceptance, and action: an emerging framework for understanding AIDS stigmatizing attitudes among community leaders in Barbados. AIDS Patient Care STDs 19(3):186-199. https://doi.org/10.1089/apc.2005.19.186 
Samuels TA, Kirton J, Guebert J (2014) Monitoring compliance with high-level commitments in health: the case of the CARICOM Summit on chronic non-communicable diseases/Controler le respect des engagements de haut niveau en matiere de sante: le cas du Sommet de la CARICOM sur les maladies chroniques non transmissibles. Bull World Health Organ 92(4):270-277

Shippee ND, Shah ND, May CR et al (2012) Cumulative complexity: a functional, patient-centered model of patient complexity can improve research and practice. J Clin Epidemiol 65(10): 1041-1051. https://doi.org/10.1016/j.jclinepi.2012.05.005

Statistics South Africa. (2014). Statistics South Africa (2014): provincial profile Limpopo. Census 2011

World Health Organization (2011) Progress report 2011: global HIV/AIDS response. WHO, Geneva

Open Access This chapter is licensed under the terms of the Creative Commons Attribution 4.0 International License (http://creativecommons.org/licenses/by/4.0/), which permits use, sharing, adaptation, distribution and reproduction in any medium or format, as long as you give appropriate credit to the original author(s) and the source, provide a link to the Creative Commons license and indicate if changes were made.

The images or other third party material in this chapter are included in the chapter's Creative Commons license, unless indicated otherwise in a credit line to the material. If material is not included in the chapter's Creative Commons license and your intended use is not permitted by statutory regulation or exceeds the permitted use, you will need to obtain permission directly from the copyright holder. 